\title{
Paternal monoenergetic neutron exposure results in abnormal sperm, and embryonal lethality and transgenerational tumorigenesis in mouse $F_{1}$ offspring
}

\author{
HIROMITSU WATANABE ${ }^{1}$, MEGUMI TOYOSHIMA ${ }^{1}$, MASAYORI ISHIKAWA $^{2}$ and KENJI KAMIYA ${ }^{1}$ \\ ${ }^{1}$ Department of Experimental Oncology, Research Institute for Radiation Biology and Medicine, Hiroshima University, \\ Kasumi 1-2-3, Minami-ku, Hiroshima 734-8553; ${ }^{2}$ Department of Molecular Trace Radiation Medicine, \\ Hokkaido University Hospital, North 15 West 7, Kita-ku, Sapporo 060-8648, Japan
}

Received December 2, 2009; Accepted January 28, 2010

DOI: 10.3892/or_00000771

\begin{abstract}
Experiments were conducted to assay whether monoenergetic neutron-induced genetic damage in parental germline cells can give rise to development of cancer in the offspring. Seven-week-old $\mathrm{C} 3 \mathrm{H}$ male mice were irradiated with monoenergetic neutrons with energy levels of 0.2 or $0.6 \mathrm{MeV}$ at doses of $0,50,100$ or $200 \mathrm{cGy}$. Two weeks after irradiation, when the male mice showed an increased incidence of sperm abnormalities, they were mated with virgin 9-week-old C57BL females. Litter size was decreased and embryo lethalities were increased in a dose-dependent manner. Furthermore, tumor incidence in male offspring born to male mice irradiated with 25 or $50 \mathrm{cGy}$ at $0.6 \mathrm{MeV}$ showed a tendency for increase as compared to the non-irradiated group value. Liver tumors in the 50 cGy group were significantly increased $(\mathrm{P}=0.03)$. It is concluded that the increased hepatic tumor risk in the $\mathrm{F}_{1}$ generation may have been caused by genetic transmission of some hepatoma-associated trait(s) induced by monoenergetic neutron irradiation.
\end{abstract}

\section{Introduction}

There is now a wealth of information on the transmission of tumor-related genetic traits through germ cells from parents to offspring and research has been performed to address this question not only in man but also experimental animals (1-3). The possible importance of such genetic transmission is evidenced by the finding of increased risk of leukemia and non-Hodgkin lymphoma in children of workers at the Sellafield nuclear plant and in the West Berkshire and North

Correspondence to: Dr Hiromitsu Watanabe, Department of Experimental Oncology, Research Institute for Radiation Biology and Medicine, Hiroshima University, 1-2-3 Kasumi, Minami-ku, Hiroshima 734-8553, Japan

E-mail: tonko@hiroshima-u.ac.jp

Key words: monoenergetic neutrons, mouse, paternal exposure, offspring, tumorigenesis
Hampshine nuclear industries (4). Furthermore, experimental evidence for germinal transmission of cancer-related genetic damage has been obtained after parental exposure to ethylnitrosourea (5), X-rays and urethane (6) and neutron irradiation (7-9).

In order to study the radiobiological effects of neutron, the Hiroshima University Radiobiological Research Accelerator (HIRRAC) can be operated under conditions of high proton beam currents of $1 \mathrm{~mA}$ and acceleration voltages up to $3 \mathrm{MeV}$. The biological effects of monoenergetic neutrons are of particular interest to basic science and radiation protection (10). Concern is reflected in in vitro assays (11-17) as well as in vivo studies (18). To our knowledge, however, there has been relatively little work on the genetic effects of monoenergetic neutrons at various energy levels using in vivo systems.

Specifications for biological irradiation are presented in terms of monoenergetic beam conditions, dose rates and deposited energy spectra. High dose rates of monoenergetic neutron fields are useful for studying the neutron energy dependency of biological effects, and also for other radiobiology studies on the basic mechanisms of the effects of neutrons. Monoenergetic neutrons which have a narrow neutron spectrum are the most useful, therefore they were chosen for the present study of whether irradiation-induced genetic damage can be passed to the offspring, causing embryonic lethality and tumor development in the $F_{1}$ generation.

\section{Materials and methods}

Animals. COBOS male C3H/HeNCrj and female C57BL/ 6NCrj mice were purchased from Charles River Japan, Inc. (Hino, Japan) and housed in autoclaved cages on sterile wood chips, in a room with controlled temperature $\left(24 \pm 2^{\circ} \mathrm{C}\right)$, humidity $(55 \pm 10 \%)$ and a regular 12-h light, 12-h dark cycle, under the guidelines set forth in the 'Guide for the Care and Use of Laboratory Animals' established by Hiroshima University. They were fed a commercial diet MF (Oriental Yeast Co., Ltd., Tokyo, Japan) and were provided with normal tap water ad libitum. All experiments used the same lot of animals. 
Table I. Body, testis, epididymis weights and abnormal sperm induced 3 weeks after monoenegic neutron.

\begin{tabular}{|c|c|c|c|c|c|c|}
\hline & BW & Testis & Epididymis & Testis/BW & Epi/bw & Sperm abnormal \\
\hline 0 cGy & $28.9 \pm 1.9$ & $0.17 \pm 0.02$ & $0.063 \pm 0.006$ & $6.01 \pm 0.76$ & $2.21 \pm 0.20$ & $1.56 \pm 0.76$ \\
\hline \multicolumn{7}{|l|}{$0.2 \mathrm{MeV}$} \\
\hline $12.5 \mathrm{cGy}$ & $27.9 \pm 1.0$ & $0.13 \pm 0.01^{\mathrm{a}}$ & $0.062 \pm 0.007$ & $4.69 \pm 0.47^{\mathrm{a}}$ & $2.22 \pm 0.22$ & $0.96 \pm 0.33$ \\
\hline $25 \mathrm{cGy}$ & $28.0 \pm 1.0$ & $0.11 \pm 0.02^{\mathrm{a}}$ & $0.056 \pm 0.004^{\mathrm{a}}$ & $3.80 \pm 0.55^{\mathrm{a}}$ & $2.01 \pm 0.16$ & $1.93 \pm 1.08$ \\
\hline $50 \mathrm{cGy}$ & $27.9 \pm 1.3$ & $0.10 \pm 0.01^{\mathrm{a}}$ & $0.054 \pm 0.003^{\mathrm{a}}$ & $3.46 \pm 0.17^{\mathrm{a}}$ & $1.95 \pm 0.12^{\mathrm{b}}$ & $1.92 \pm 1.18$ \\
\hline \multirow[t]{4}{*}{$100 \mathrm{cGy}$} & $26.7 \pm 1.2^{\mathrm{a}}$ & $0.08 \pm 0.01^{\mathrm{a}}$ & $0.053 \pm 0.005^{\mathrm{a}}$ & $2.92 \pm 0.34^{\mathrm{a}}$ & $1.97 \pm 0.23^{\mathrm{b}}$ & $4.06 \pm 1.16^{\mathrm{a}}$ \\
\hline & & $Y=-0.078 X+0.14$ & & $Y=-0.026 X+5.2$ & & $Y=0.026 X+1.07$ \\
\hline & & $\mathrm{r}^{2}=-0.90$ & & $\mathrm{r}^{2}=-0.89$ & & $r^{2}=0.92$ \\
\hline & & $\mathrm{P}<0.05$ & & $\mathrm{P}<0.05$ & & $\mathrm{P}<0.05$ \\
\hline \multicolumn{7}{|l|}{$0.6 \mathrm{MeV}$} \\
\hline $12.5 \mathrm{cGy}$ & $27.7 \pm 1.1$ & $0.13 \pm 0.01^{\mathrm{a}}$ & $0.061 \pm 0.006$ & $4.70 \pm 0.35^{\mathrm{a}}$ & $2.19 \pm 0.20$ & $1.50 \pm 0.65$ \\
\hline $25 \mathrm{cGy}$ & $29.3 \pm 1.9$ & $0.11 \pm 0.01^{\mathrm{a}}$ & $0.061 \pm 0.027$ & $3.66 \pm 0.37^{\mathrm{a}}$ & $2.10 \pm 0.21$ & $2.36 \pm 1.66$ \\
\hline $50 \mathrm{cGy}$ & $28.0 \pm 1.3$ & $0.09 \pm 0.01^{\mathrm{a}}$ & $0.057 \pm 0.002^{\mathrm{b}}$ & $3.36 \pm 0.33^{\mathrm{a}}$ & $2.02 \pm 0.09$ & $3.07 \pm 1.43^{b}$ \\
\hline \multirow[t]{4}{*}{100 cGy } & $27.5 \pm 1.1^{\mathrm{b}}$ & $0.08 \pm 0.01^{\mathrm{a}}$ & $0.055 \pm 0.005^{\mathrm{a}}$ & $2.91 \pm 0.18^{\mathrm{a}}$ & $1.98 \pm 0.20^{\mathrm{b}}$ & $6.18 \pm 1.07^{\mathrm{a}, \mathrm{c}}$ \\
\hline & & $Y=-0.078 X+0.14$ & & $Y=-0.026 X+5.1$ & & $Y=0.048 X+1.14$ \\
\hline & & $r^{2}=-0.86$ & & $\mathrm{r}^{2}=-0.84$ & & $\mathrm{r}^{2}=0.98$ \\
\hline & & & & & & $\mathrm{P}<0.01$ \\
\hline
\end{tabular}

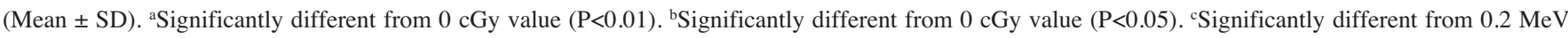
100 cGy value $(\mathrm{P}<0.01)$.

Monoenergetic neutron irradiation. Neutron sources in this study was produced by Hiroshima University Radiobiological Research Accelerator (HIRRAC) as described previously (18). The HIRRAC can generate various monoenergetic neutrons using ${ }^{7} \mathrm{Li}(\mathrm{p}, \mathrm{n})^{7} \mathrm{Be}$ reaction with maximum accelerated voltage of $3 \mathrm{MV}$.

The absorbed doses were evaluated using paired ionization chambers IC-17 ATW (FWT, Inc., Goleta, CA, USA) and IC-17G (model GM539, FWT, Inc.). The IC-17ATW, which is made of tissue equivalent materials and filled with propanebase tissue equivalent gas, can measure the sum of neutron and $\gamma$-ray dose. The IC- $17 \mathrm{G}$, which is made of carbon and filled with carbon dioxide gas, can measure $\gamma$-ray dose with a few neutron dose contributions. Using these chambers, separate dose of neutron and $\gamma$-ray can be evaluated. The $\gamma$ ray contamination was estimated $<3 \%$ of neutron dose when using 10- $\mu$ m-thick lithium targets.

Each mouse was put into a box $(3 \mathrm{~cm} \times 3 \mathrm{~cm} \times 5 \mathrm{~cm})$ and 5 mice were located $20 \mathrm{~cm}$ away from target plane and $10 \mathrm{~cm}$ away from beam axis, which means that the mice were placed at 30 degrees direction position.

In order to uniform individual neutron doses, mice were rotated with a speed of $1 \mathrm{rpm}$. Groups of 5 mice were exposed by monoernergetic neutrons in 0.20 and $0.6 \mathrm{MeV}$ (dose $50 \mathrm{cGy}$, dose rate $0.5 \mathrm{cGy} / \mathrm{min}$ ) without anesthesia. The accelerated voltages for their neutron energy were 2.0 and 2.37 MV, respectively.

Experiments. One hundred and ten male mice received a single whole body exposure to monoernergetic neutrons with energy levels of 0.2 or $0.6 \mathrm{MeV}$ at doses of $0,25,50,100$ or
200 cGy. Two weeks (spermatid stage) after irradiation, the males were mated with 3 non-irradiated 9-week-old C57BL female mice for a week, and retired males were then sacrificed. Testes were minced in saline and filtered and sperm were stained with Giemsa solution to allow the numbers of normal and abnormal sperm to be counted (19).

A total of 47 successfully mated females in one group were sacrificed 18 days after fertilization and the numbers of surviving and dead embryos were counted. In the remainder, offspring were obtained, the ratio of surviving pups was determined 1 week after birth, and the $\mathrm{F}_{1}$ mice were maintained until 13.5 months of age.

Pathology. All animals were regularly observed on a daily basis and weighed once a month. At the time of necropsy, full autopsies were carried out under ether anesthesia, and body weights and various organ weights were determined. The number and size of liver tumor nodules were also measured and diseases of the liver and other organs including neoplastic changes were diagnosed by routine histological examination.

Statistical analysis. The significance of differences in numerical data was determined using the $\chi^{2}$, Student's t-tests and the Dunnett method for multiple comparisons using logarithmic transformation.

\section{Results}

Changes in body and organ weights and appearance of abnormal sperm in the irradiated mice. Body weights of 
Table II. Females mice used.

\begin{tabular}{|c|c|c|c|c|c|c|}
\hline \multirow[b]{3}{*}{0 cGy } & \multirow{3}{*}{$\begin{array}{c}\text { Used females } \\
37\end{array}$} & \multirow[b]{2}{*}{ Non-pregnancy } & \multicolumn{4}{|c|}{ Pregnancy } \\
\hline & & & \multirow{2}{*}{$\begin{array}{c}\text { Used for embryo lethality } \\
4\end{array}$} & Non-nursing & Nursing (\%) & \multirow{2}{*}{$\begin{array}{r}\text { Tota } \\
18\end{array}$} \\
\hline & & $15 \quad(41)$ & & $1 \quad(6)$ & $17 \quad(94)$ & \\
\hline \multicolumn{7}{|l|}{$0.2 \mathrm{MeV}$} \\
\hline $12.5 \mathrm{cGy}$ & 20 & $6 \quad(30)$ & 8 & 0 & $6(100)$ & 6 \\
\hline $25 \mathrm{cGy}$ & 15 & $5 \quad(33)$ & 3 & 1 (14) & $6 \quad(86)$ & 7 \\
\hline $50 \mathrm{cGy}$ & 16 & 3 (19) & 5 & 0 & $8(100)$ & 8 \\
\hline 100 cGy & 16 & $5 \quad(31)$ & 4 & 2 (29) & $5 \quad(71)$ & 7 \\
\hline \multicolumn{7}{|l|}{$0.6 \mathrm{MeV}$} \\
\hline $12.5 \mathrm{cGy}$ & 19 & $7 \quad(37)$ & 7 & $1 \quad(20)$ & $4 \quad(80)$ & 5 \\
\hline $25 \mathrm{cGy}$ & 20 & $4 \quad(20)$ & 8 & 1 (13) & $7 \quad(88)$ & 8 \\
\hline $50 \mathrm{cGy}$ & 15 & $5 \quad(33)$ & 3 & 0 & $7(100)$ & 7 \\
\hline $100 \mathrm{cGy}$ & 37 & $16 \quad(43)$ & 5 & 11 (65) & $6 \quad(35)$ & 17 \\
\hline
\end{tabular}

100 cGy irradiated males with both energies were significantly decreased as compared with non-irradiated controls.

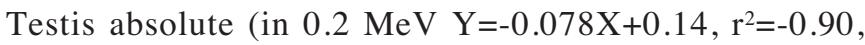
$\mathrm{P}<0.05$; in $\left.0.6 \mathrm{MeV} \mathrm{Y}=-0.078 \mathrm{X}+0.14, \mathrm{r}^{2}=-0.86\right)$ and relative weights (in $0.2 \mathrm{MeV} \mathrm{Y}=-0.026 \mathrm{X}+5.2, \mathrm{r}^{2}=-0.89, \mathrm{P}<0.05$ ); in $\left.0.6 \mathrm{MeV} \mathrm{Y}=-0.26 \mathrm{X}+5.1, \mathrm{r}^{2}=-0.84\right)$ were also decreased linearly. The epididymis weights were decreased. Ratios of abnormal sperm were increased and with $100 \mathrm{cGy}$ at $0.6 \mathrm{MeV}$ the value was significantly greater than with $0.2 \mathrm{MeV}$ (Table I) $\left(0.2 \mathrm{MeV} \mathrm{Y}=0.026 \mathrm{X}+1.07, \mathrm{r}^{2}=0.92, \mathrm{P}<0.05\right.$; in $0.6 \mathrm{MeV}$ $\left.\mathrm{Y}=0.048 \mathrm{X}+1.14, \mathrm{r}^{2}=0.98, \mathrm{P}<0.01\right)$.

Survival of embryos. Data for used female mice are shown in Table II. Non-pregnant females accounted for 19-43\%. Numbers of implantations per mouse were decreased in a dose-dependent manner (Table III 0.2 MeV Y=-0.035X+8.78, $\mathrm{r}^{2}=-0.91, \mathrm{P}<0.05$; in $0.6 \mathrm{MeV} \mathrm{Y}=-0.034 \mathrm{X}+93, \mathrm{r}^{2}=-0.90$, $\mathrm{P}<0.05)$. Numbers of total embryos in $100 \mathrm{cGy}$ with both energy levels were significantly decreased as compared with other dose groups (Table III). Numbers of surviving embryos were significantly lower with 100 cGy irradiation with the average numbers of surviving embryos per mother were decreased in a dose-dependent manner (in $0.2 \mathrm{MeV}$ $\mathrm{Y}=-0.058 \mathrm{X}+7.5, \mathrm{r}^{2}=-0.99, \mathrm{P}<0.01$; in $0.6 \mathrm{MeV} \mathrm{Y}=-0.045 \mathrm{X}+$ $\left.7.6, \mathrm{r}^{2}=-0.97, \mathrm{P}<0.01\right)$. Conversely, lethality increased with the dose (in $0.2 \mathrm{MeV} \mathrm{Y}=0.02 \mathrm{X}+1.57, \mathrm{r}^{2}=0.91, \mathrm{P}<0.05$; in $\left.0.6 \mathrm{MeV} \mathrm{Y}=0.01 \mathrm{X}+1.71, \mathrm{r}^{2}=0.65\right)$.

Birth rate and offspring nursing rate. Data for non-nursing mothers are given in Table II. The number was increased with $100 \mathrm{cGy}$ at the $0.6 \mathrm{MeV}$ energy level.

Offspring from mating two weeks after irradiation. Data for litter size and sex ratios are given in Table IV. Mean offspring number per mother was decreased dose-dependently at the $0.2 \mathrm{MeV}$ energy level (total pups $\mathrm{Y}=-0.05 \mathrm{X}+8.3, \mathrm{r}^{2}=-0.99$, $\mathrm{P}<0.01$; male $\mathrm{Y}=-0.03 \mathrm{X}+4.0, \mathrm{r}^{2}=-0.96, \mathrm{P}<0.01$; female $\mathrm{Y}=-0.024 \mathrm{X}+4.2, \mathrm{r}^{2}=-0.94, \mathrm{P}<0.05$ ) and with $0.6 \mathrm{MeV}$ (total
Table III. Mean survival data for embryos.

\begin{tabular}{lccc}
\hline Group & Survival & Lethal & Total \\
\hline $0 \mathrm{cGy}$ & $7.50 \pm 1.00^{\mathrm{a}, \mathrm{c}}$ & $1.50 \pm 1.00$ & $9.00 \pm 1.15 \mathrm{a}^{\mathrm{a}, \mathrm{c}}$ \\
$0.2 \mathrm{MeV}$ & & & \\
$12.5 \mathrm{cGy}$ & $6.50 \pm 1.20^{\mathrm{a}, \mathrm{c}}$ & $1.38 \pm 1.30$ & $7.88 \pm 0.99^{\mathrm{a}, \mathrm{c}}$ \\
$25 \mathrm{cGy}$ & $5.00 \pm 2.00^{\mathrm{b}}$ & $2.67 \pm 2.52$ & $7.67 \pm 0.58^{\mathrm{b}}$ \\
$50 \mathrm{cGy}$ & $5.20 \pm 2.28^{\mathrm{a}}$ & $2.60 \pm 2.07$ & $7.80 \pm 1.30^{\mathrm{a}, \mathrm{d}}$ \\
$100 \mathrm{cGy}$ & $1.50 \pm 1.29^{\mathrm{e}}$ & $3.50 \pm 1.29$ & $5.00 \pm 2.00^{\mathrm{e}}$ \\
& $\mathrm{Y}=-0.058 \mathrm{X}+7.5$ & $\mathrm{Y}=0.02 \mathrm{X}+1.57$ & $\mathrm{Y}=-0.035 \mathrm{X}+8.78$ \\
& $\mathrm{r}^{2}=-0.99, \mathrm{P}<0.01$ & $\mathrm{r}^{2}=0.91, \mathrm{P}<0.05$ & $\mathrm{r}^{2}=-0.91, \mathrm{P}<.0 .05$ \\
$0.6 \mathrm{MeV}$ & & & \\
$12.5 \mathrm{cGy}$ & $7.43 \pm 1.72^{\mathrm{a}, \mathrm{c}}$ & $1.43 \pm 1.27$ & $8.86 \pm 1.57^{\mathrm{a}, \mathrm{c}}$ \\
$25 \mathrm{cGy}$ & $5.75 \pm 2.05^{\mathrm{a}, \mathrm{d}}$ & $2.23 \pm 1.30$ & $8.13 \pm 1.36^{\mathrm{a}, \mathrm{c}}$ \\
$50 \mathrm{cGy}$ & $5.67 \pm 0.58^{\mathrm{a}}$ & $3.00 \pm 2.00$ & $8.67 \pm 1.53^{\mathrm{a}, \mathrm{c}}$ \\
$100 \mathrm{cGy}$ & $3.00 \pm 1.22^{\mathrm{e}}$ & $2.40 \pm 1.52$ & $5.40 \pm 0.55^{\mathrm{e}}$ \\
& $\mathrm{Y}=-0.045 \mathrm{X}+7.6$ & $\mathrm{Y}=0.01 \mathrm{X}+1.71$ & $\mathrm{Y}=-0.034 \mathrm{X}+9.3$ \\
& $\mathrm{r}^{2}=0.97, \mathrm{P}<0.01$ & $\mathrm{r}^{2}=0.65$ & $\mathrm{r}^{2}=-0.90, \mathrm{P}<0.05$
\end{tabular}

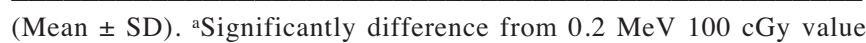
$(\mathrm{P}<0.01)$. ${ }^{\mathrm{b}}$ Significantly difference from $0.2 \mathrm{MeV} 100 \mathrm{cGy}$ value $(\mathrm{P}<0.05)$. ${ }^{\mathrm{c}}$ Significantly difference from $0.6 \mathrm{MeV} 100 \mathrm{cGy}$ value $(\mathrm{P}<0.01)$. ${ }^{\mathrm{d}}$ Significantly difference from $0.6 \mathrm{MeV} 100 \mathrm{cGy}$ value $(\mathrm{P}<0.05)$. ${ }^{\text {eS Significantly }}$ difference from $0 \mathrm{cGy}$ value $(\mathrm{P}<0.01)$.

$\mathrm{Y}=-0.06 \mathrm{X}+8.0, \mathrm{r}^{2}=-0.97, \mathrm{P}<0.01$; female $\mathrm{Y}=-0.04 \mathrm{X}+4.4$, $\left.\mathrm{r}^{2}=-0.86\right)$ except in males $\left(\mathrm{Y}=-0.01 \mathrm{X}+2.8, \mathrm{r}^{2}=-0.57\right)$. The sex ratio at $0.2 \mathrm{MeV}$ was about $50: 50$ but at $0.6 \mathrm{MeV}$ differed with $12.5 \mathrm{cGy}$. In the long-term study, total number of offspring with $100 \mathrm{cGy}$ at both energy levels was small.

Sequential assessment showed significant increase in body weights with $50 \mathrm{cGy}$ at $0.2 \mathrm{MeV}$ during 4-7 months and with $50 \mathrm{cGy}$ at $0.6 \mathrm{MeV}$ during to 12 months in males as 
Table IV. Sex ratio after birth and effective animals.

\begin{tabular}{|c|c|c|c|c|c|c|}
\hline \multirow[b]{2}{*}{ Group } & \multicolumn{3}{|c|}{ Sex ratio } & \multicolumn{3}{|c|}{ No. of animals } \\
\hline & Total & Male & Female & Total & Male $(\%)$ & Female $(\%)$ \\
\hline 0 cGy & $8.53 \pm 1.37$ & $4.53 \pm 1.37$ & $3.88 \pm 1.73$ & 138 & $74(54)$ & $64(46)$ \\
\hline \multicolumn{7}{|l|}{$0.2 \mathrm{MeV}$} \\
\hline $12.5 \mathrm{cGy}$ & $7.33 \pm 1.51$ & $3.33 \pm 1.63$ & $4.00 \pm 1.26$ & 47 & $24(51)$ & $23(49)$ \\
\hline $25 \mathrm{cGy}$ & $7.33 \pm 1.21$ & $3.17 \pm 0.98$ & $4.17 \pm 0.75$ & 43 & $21(49)$ & $22(51)$ \\
\hline $50 \mathrm{cGy}$ & $5.38 \pm 1.85^{\mathrm{a}}$ & $2.63 \pm 0.92^{\mathrm{a}}$ & $2.75 \pm 1.28$ & 40 & $20(50)$ & $20(50)$ \\
\hline \multirow[t]{3}{*}{100 cGy } & $3.20 \pm 0.84^{\mathrm{a}}$ & $1.40 \pm 0.89^{\mathrm{a}}$ & $1.80 \pm 0.84^{\mathrm{b}}$ & 16 & $7(44)$ & $9(56)$ \\
\hline & $Y=-0.05 X+8.3$ & $Y=-0.03 X+4.0$ & $Y=-0.024 X+4.22$ & & & \\
\hline & $\mathrm{r}^{2}=-0.99, \mathrm{P}<0.01$ & $\mathrm{r}^{2}=-0.96, \mathrm{P}<0.01$ & $\mathrm{r}^{2}=-0.94, \mathrm{P}<0.05$ & & & \\
\hline \multicolumn{7}{|l|}{$0.6 \mathrm{MeV}$} \\
\hline $12.5 \mathrm{cGy}$ & $7.50 \pm 1.9$ & $2.50 \pm 2.38^{b}$ & $5.00 \pm 2.16$ & 29 & $10(34)$ & $19(66)$ \\
\hline $25 \mathrm{cGy}$ & $6.00 \pm 1.63^{\mathrm{a}}$ & $2.29 \pm 1.25^{\mathrm{a}}$ & $3.71 \pm 1.60$ & 38 & $16(42)$ & $22(58)$ \\
\hline $50 \mathrm{cGy}$ & $4.43 \pm 0.98^{\mathrm{a}}$ & $3.14 \pm 1.21$ & $1.29 \pm 1.11^{\mathrm{a}}$ & 42 & $22(52)$ & $20(48)$ \\
\hline \multirow[t]{3}{*}{100 cGy } & $2.50 \pm 1.22^{\mathrm{a}}$ & $1.50 \pm 0.84^{\mathrm{a}}$ & $1.00 \pm 0.63^{\mathrm{a}}$ & 17 & $8(47)$ & $9(53)$ \\
\hline & $Y=-0.06 X+8.0$ & $Y=-0.01 X+2.8$ & $Y=-0.04 X+4.4$ & & & \\
\hline & $\mathrm{r}^{2}=-0.97, \mathrm{P}<0.01$ & $\mathrm{r}^{2}=-0.57$ & $r^{2}=-0.86$ & & & \\
\hline
\end{tabular}

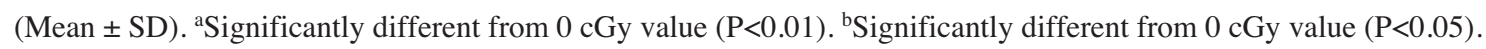

Table V. Body weights of $F_{1}$ male mice.

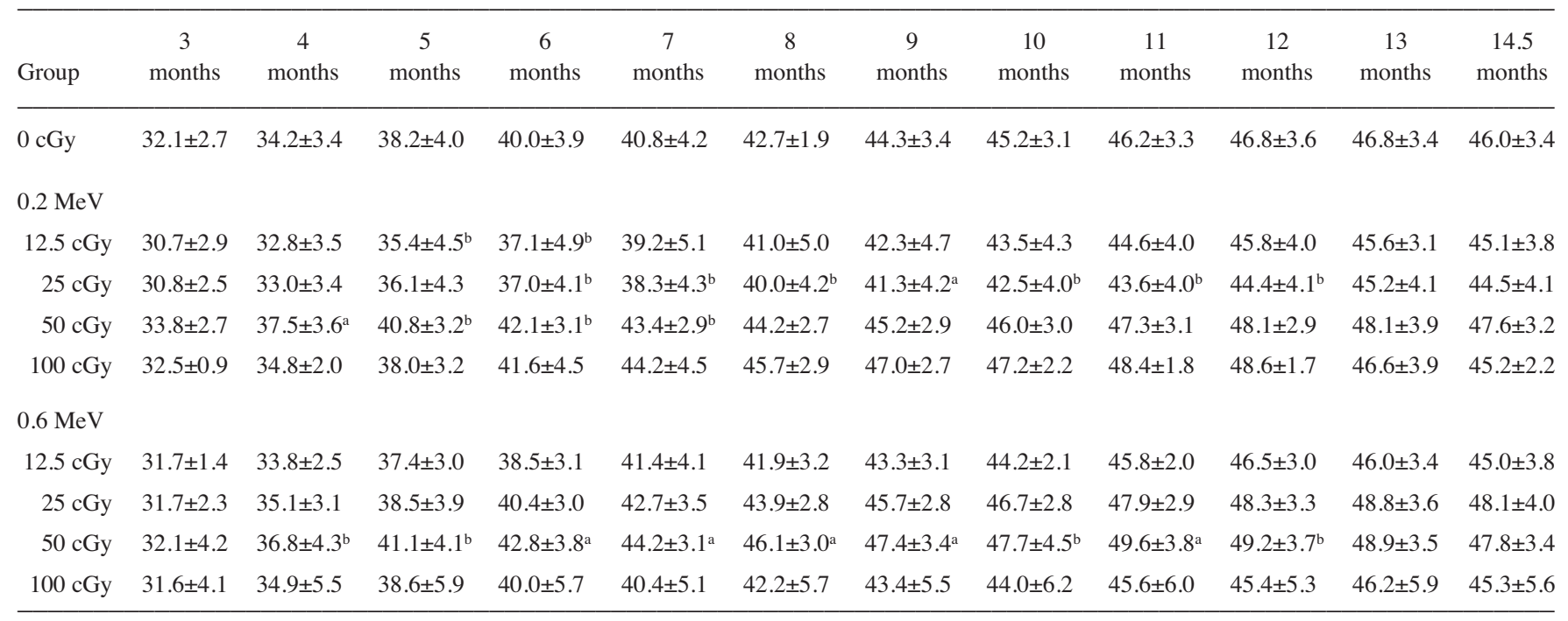

(Mean $\pm \mathrm{SD})$. ${ }^{\mathrm{a} S i g n i f i c a n t l y ~ d i f f e r e n t ~ f r o m ~} 0 \mathrm{cGy}$ value $(\mathrm{P}<0.01)$. ${ }^{\mathrm{b}}$ Significantly different from 0 cGy value $(\mathrm{P}<0.05)$.

compared to control males (Table V), whereas significantly decrease was evident with $25 \mathrm{cGy}$ at $0.2 \mathrm{MeV}$. Female body weights were significantly heavier than for controls with $50 \mathrm{cGy}$ at $0.2 \mathrm{MeV}$ from 3 to 6 months, with $100 \mathrm{cGy}$ at $0.2 \mathrm{MeV}$ during the whole experiment, with $25 \mathrm{cGy}$ at $0.6 \mathrm{MeV}$ from 5 to 12 months, and with $50 \mathrm{cGy}$ at $0.6 \mathrm{MeV}$ from 5 to 13.5 months, whereas with $25 \mathrm{cGy}$ they were decreased from 8 to 13.5 months as compared with control values (Table VI).
At autopsy, body weights of male $\mathrm{F}_{1}$ mice of the $0.2 \mathrm{MeV}$ energy level groups were not significantly altered (Table VII). Testis weights with 100 cGy were significantly lower than the non-irradiated group whereas adrenals were heavier. Relative testis weights (organ weight/body weight x1000) with 50 and 100 cGy were also significantly decreased as compared with the non-irradiated group and again adrenal values were increased (Table VIII). 
Table VI. Body weights of $\mathrm{F}_{1}$ female mice.

\begin{tabular}{|c|c|c|c|c|c|c|c|c|c|c|c|c|}
\hline Group & $\begin{array}{c}3 \\
\text { months }\end{array}$ & $\begin{array}{c}4 \\
\text { months }\end{array}$ & $\begin{array}{c}5 \\
\text { months }\end{array}$ & $\begin{array}{c}6 \\
\text { months }\end{array}$ & $\begin{array}{c}7 \\
\text { months }\end{array}$ & $\begin{array}{c}8 \\
\text { months }\end{array}$ & $\begin{array}{c}9 \\
\text { months }\end{array}$ & $\begin{array}{c}10 \\
\text { months }\end{array}$ & $\begin{array}{c}11 \\
\text { months }\end{array}$ & $\begin{array}{c}12 \\
\text { months }\end{array}$ & $\begin{array}{c}13 \\
\text { months }\end{array}$ & $\begin{array}{c}14.5 \\
\text { months }\end{array}$ \\
\hline \multicolumn{13}{|l|}{$0.2 \mathrm{MeV}$} \\
\hline $12.5 \mathrm{cGy}$ & $24.7 \pm 2.0$ & $26.0 \pm 2.3$ & $27.9 \pm 2.9$ & $30.2 \pm 3.3$ & $31.1 \pm 3.6$ & $33.9 \pm 4.3$ & $35.8 \pm 4.4$ & $38.0 \pm 5.1$ & $41.1 \pm 6.2$ & $41.9 \pm 6.8$ & $42.8 \pm 7.2$ & $42.8 \pm 7.1$ \\
\hline $25 \mathrm{cGy}$ & $24.1 \pm 1.5$ & $25.4 \pm 2.1$ & $27.1 \pm 2.9$ & $28.3 \pm 2.7$ & $30.0 \pm 2.8$ & $30.7 \pm 3.1^{\mathrm{b}}$ & $32.7 \pm 3.6^{\mathrm{b}}$ & $33.6 \pm 3.7^{b}$ & $36.5 \pm 4.2^{\mathrm{b}}$ & $37.9 \pm 4.4^{\mathrm{a}}$ & $37.6 \pm 4.7^{\mathrm{a}}$ & $38.3 \pm 4.6^{\mathrm{b}}$ \\
\hline 50 cGy & $26.0 \pm 2.1^{\mathrm{b}}$ & $28.6 \pm 4.2^{\mathrm{a}}$ & $30.0 \pm 3.6^{\mathrm{a}}$ & $32.6 \pm 4.4^{b}$ & $34.3 \pm 5.2$ & $36.4 \pm 5.8$ & $38.2 \pm 5.4$ & $40.3 \pm 6.7$ & $43.7 \pm 6.0$ & $46.2 \pm 5.8$ & $47.1 \pm 5.6$ & $44.4 \pm 9.3$ \\
\hline 100 cGy & $27.8 \pm 3.0^{\mathrm{a}}$ & $31.0 \pm 3.2^{\mathrm{a}}$ & $33.9 \pm 3.9^{\mathrm{a}}$ & $36.6 \pm 3.3^{\mathrm{a}}$ & $38.5 \pm 5.2^{\mathrm{a}}$ & $40.3 \pm 4.2^{\mathrm{a}}$ & $42.3 \pm 4.1^{\mathrm{a}}$ & $44.0 \pm 3.4^{\mathrm{a}}$ & $47.4 \pm 3.7^{\mathrm{a}}$ & $49.9 \pm 2.8^{a}$ & $50.0 \pm 3.0^{\mathrm{b}}$ & $48.9 \pm 3.5^{b}$ \\
\hline \multicolumn{13}{|l|}{$0.6 \mathrm{MeV}$} \\
\hline $12.5 \mathrm{cGy}$ & $24.5 \pm 1.8$ & $25.8 \pm 3.2$ & $28.4 \pm 3.6$ & $29.7 \pm 4.2$ & $31.5 \pm 4.3$ & $35.1 \pm 6.1$ & $38.3 \pm 6.3$ & $38.3 \pm 6.7$ & $40.7 \pm 6.9$ & $41.0 \pm 6.7$ & $40.7 \pm 6.9$ & $39.6 \pm 6.9$ \\
\hline 25 cGy & $24.6 \pm 2.3$ & $27.1 \pm 2.9$ & $31.3 \pm 4.9^{\mathrm{a}}$ & $33.2 \pm 5.2^{\mathrm{a}}$ & $35.4 \pm 5.2^{\mathrm{a}}$ & $38.4 \pm 6.0^{\mathrm{a}}$ & $40.9 \pm 6.0^{\mathrm{a}}$ & $42.3 \pm 6.1^{\mathrm{a}}$ & $45.3 \pm 6.3^{\mathrm{a}}$ & $46.4 \pm 6.2^{\mathrm{b}}$ & $45.8 \pm 10.9$ & $47.4 \pm 6.6$ \\
\hline 100 cGy & $26.5 \pm 2.5^{\mathrm{b}}$ & $24.8 \pm 11.4$ & $31.9 \pm 6.0^{\mathrm{a}}$ & $34.0 \pm 7.1^{\mathrm{b}}$ & $35.7 \pm 7.4$ & $38.0 \pm 7.4$ & $40.0 \pm 8.4$ & $42.2 \pm 8.8$ & $45.7 \pm 9.0$ & $46.0 \pm 8.1$ & $47.3 \pm 9.9$ & $46.3 \pm 9.1$ \\
\hline
\end{tabular}

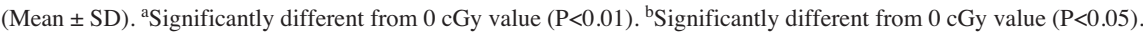

Table VII. Body and organ weight for $\mathrm{F}_{1}$ male.

\begin{tabular}{|c|c|c|c|c|c|c|}
\hline Group & Body weight & Liver & Kidney & Testis & Adrenal & Spleen \\
\hline 0 cGy & $46.0 \pm 3.4$ & $2.15 \pm 0.36$ & $0.61 \pm 0.07$ & $0.21 \pm 0.01$ & $0.007 \pm 0.002$ & $0.11 \pm 0.03$ \\
\hline \multicolumn{7}{|l|}{$0.2 \mathrm{MeV}$} \\
\hline 12.5 cGy & $45.1 \pm 3.8$ & $2.20 \pm 0.33$ & $0.62 \pm 0.07$ & $0.20 \pm 0.02$ & $0.006 \pm 0.001$ & $0.10 \pm 0.03$ \\
\hline 25 cGy & $44.5 \pm 4.1$ & $2.05 \pm 0.28$ & $0.59 \pm 0.08$ & $0.20 \pm 0.03$ & $0.006 \pm 0.001$ & $0.10 \pm 0.02$ \\
\hline 50 cGy & $47.6 \pm 3.2$ & $2.30 \pm 0.36$ & $0.63 \pm 0.09$ & $0.20 \pm 0.002$ & $0.007 \pm 0.002$ & $0.12 \pm 0.06$ \\
\hline 100 cGy & $45.2 \pm 2.2$ & $2.19 \pm 0.38$ & $0.58 \pm 0.04$ & $0.18 \pm 0.05^{\mathrm{b}}$ & $0.026 \pm 0.042^{\mathrm{a}}$ & $0.13 \pm 0.12$ \\
\hline \multicolumn{7}{|l|}{$0.6 \mathrm{MeV}$} \\
\hline 12.5 cGy & $45.0 \pm 3.8$ & $2.00 \pm 0.24$ & $0.62 \pm 0.09$ & $0.21 \pm 0.01$ & $0.007 \pm 0.002$ & $0.10 \pm 0.01$ \\
\hline 25 cGy & $48.1 \pm 4.0$ & $2.52 \pm 0.58$ & $0.69 \pm 0.07^{\mathrm{a}}$ & $0.21 \pm 0.02$ & $0.009 \pm 0.002$ & $0.12 \pm 0.05$ \\
\hline 50 cGy & $47.8 \pm 3.4$ & $2.27 \pm 0.38$ & $0.61 \pm 0.05$ & $0.19 \pm 0.05^{\mathrm{b}}$ & $0.008 \pm 0.001$ & $0.12 \pm 0.03$ \\
\hline $100 \mathrm{cGy}$ & $45.3 \pm 5.6$ & $2.22 \pm 0.63$ & $0.57 \pm 0.12$ & $0.18 \pm 0.03^{b}$ & $0.008 \pm 0.003$ & $0.11 \pm 0.03$ \\
\hline
\end{tabular}

$(\mathrm{Mean} \pm \mathrm{SD}) .{ }^{\mathrm{a}}$ Significantly different from 0 cGy value $(\mathrm{P}<0.01)$. ${ }^{\mathrm{b}}$ Significantly different from 0 cGy value $(\mathrm{P}<0.05)$.

Body and kidney weights with $25 \mathrm{cGy}$ at the $0.6 \mathrm{MeV}$ energy level were increased, along with the relative liver and kidney weights in $25 \mathrm{cGy}$ were heavier than non-irradiated group but testis in 50 cGy was decreased.

Table IX summarizes data for tumors in male $\mathrm{F}_{1}$ offspring. Most lesions were liver tumors. Incidences overall were 25.7, $8.34 .8,25.0$ and $42.9 \%$ with $0,12.5,25,50$ and $100 \mathrm{cGy}$ at the $0.2 \mathrm{MeV}$ energy level, respectively, and 0, 37.5, 45.5 and $25 \%$ at $0.6 \mathrm{MeV}$. Incidences of liver tumors were 18.9 , $8.3,4.8,25.0$ and $28.6 \%$ at the $0.2 \mathrm{MeV}$ energy level, respectively, and $0,31.3,40.1(\mathrm{P}=0.03)$ and $25 \%$ at $0.6 \mathrm{MeV}$. Sizes and number of liver tumors did not significantly differ among the groups.
Female mouse body and organ weights are shown in Table X. Body weights with $25 \mathrm{cGy}$ at $0.2 \mathrm{MeV}$ were significantly decreased as compared with the non-irradiated group, and ovary and adrenal weights were significantly increased with $100 \mathrm{cGy}$ and liver and kidney weights with 25 and $50 \mathrm{cGy}$. Relative adrenal weights with $12.5 \mathrm{cGy}$ and liver with 100 cGy were significantly decreased whereas ovary values were elevated at $100 \mathrm{cGy}$ (Table XI).

Regarding incidences of tumors in females, three tumors (4.7\%, hemangioma, lymphoma and ovary) appeared in the non-irradiated group, and values were $3 / 23$ (13\%, hepatoma, lung and ovary tumors), $3 / 22$ (14\%, ovary tumor), $1 / 20$ (5\%, ovary tumor) and 0 in the $12.5,25,50$ and 100 cGy groups at 
Table VIII. Relative organ weight for $\mathrm{F}_{1}$ male mice.

\begin{tabular}{|c|c|c|c|c|c|}
\hline Group & Liver & Kidney & Testis & Adrenal & Spleen \\
\hline 0 cGy & $46.7 \pm 7.2$ & $13.3 \pm 1.1$ & $4.5 \pm 0.3$ & $0.16 \pm 0.04$ & $2.5 \pm 0.7$ \\
\hline \multicolumn{6}{|l|}{$0.2 \mathrm{MeV}$} \\
\hline $12.5 \mathrm{cGy}$ & $48.8 \pm 6.1$ & $13.8 \pm 0.9$ & $4.3 \pm 0.4$ & $0.14 \pm 0.03$ & $2.2 \pm 0.6$ \\
\hline $25 \mathrm{cGy}$ & $46.1 \pm 3.2$ & $13.3 \pm 1.4$ & $4.5 \pm 0.6$ & $0.14 \pm 0.03$ & $2.3 \pm 0.4$ \\
\hline $50 \mathrm{cGy}$ & $48.3 \pm 5.8$ & $13.3 \pm 1.4$ & $4.1 \pm 0.8^{\mathrm{b}}$ & $0.15 \pm 0.03$ & $2.5 \pm 1.3$ \\
\hline 100 cGy & $48.5 \pm 7.8$ & $12.8 \pm 1.1$ & $3.9 \pm 1.0^{\mathrm{b}}$ & $0.59 \pm 0.93^{\mathrm{a}}$ & $3.2 \pm 3.0$ \\
\hline \multicolumn{6}{|l|}{$0.6 \mathrm{MeV}$} \\
\hline $12.5 \mathrm{cGy}$ & $44.3 \pm 2.4$ & $13.7 \pm 1.7$ & $4.6 \pm 0.4$ & $0.17 \pm 0.05$ & $2.2 \pm 0.3$ \\
\hline $25 \mathrm{cGy}$ & $52.0 \pm 9.6^{\mathrm{b}}$ & $14.5 \pm 0.9^{a}$ & $4.3 \pm 0.4$ & $0.18 \pm 0.05$ & $2.5 \pm 1.1$ \\
\hline $50 \mathrm{cGy}$ & $47.2 \pm 5.7$ & $12.9 \pm 0.9$ & $4.0 \pm 1.1^{\mathrm{a}}$ & $0.17 \pm 0.03$ & $2.5 \pm 0.5$ \\
\hline $100 \mathrm{cGy}$ & $48.2 \pm 9.1$ & $12.6 \pm 1.4$ & $4.1 \pm 0.7$ & $0.17 \pm 0.05$ & $2.4 \pm 0.5$ \\
\hline
\end{tabular}

$($ Mean $\pm \mathrm{SD})$. ${ }^{\mathrm{a} S i g n i f i c a n t l y ~ d i f f e r e n t ~ f r o m ~} 0 \mathrm{cGy}$ value $(\mathrm{P}<0.01)$. ${ }^{\mathrm{b}}$ Significantly different from 0 cGy value $(\mathrm{P}<0.05)$.

Table IX. Incidence of tumors for $\mathrm{F}_{1}$ male mice.

\begin{tabular}{|c|c|c|c|c|c|c|}
\hline Group & $\begin{array}{l}\text { Effective no. } \\
\text { of animal }\end{array}$ & $\begin{array}{l}\text { Tumor bearing } \\
\text { animal }\end{array}$ & Incidence & $\begin{array}{l}\text { Liver tumor } \\
\text { size }\end{array}$ & $\begin{array}{l}\text { No. of liver tumor } \\
\text { per mouse }\end{array}$ & Other tumor \\
\hline 0 cGy & 74 & $19(25.7)$ & $14(18.9)$ & $1.59 \pm 4.13$ & $0.20 \pm 0.40$ & Lung papilloma \\
\hline \multicolumn{7}{|l|}{$0.2 \mathrm{MeV}$} \\
\hline 12.5 cGy & 24 & $2(8.3)$ & $2(8.3)$ & $1.04 \pm 3.53$ & $0.08 \pm 0.28$ & \\
\hline 25 cGy & 21 & $1 \quad(4.8)$ & $1 \quad(4.8)$ & $0.24 \pm 1.09$ & $0.05 \pm 0.22$ & \\
\hline 50 cGy & 20 & $5(25.0)$ & $5(25.0)$ & $1.57 \pm 3.45$ & $0.35 \pm 0.67$ & \\
\hline 100 cGy & 7 & $3(42.9)$ & $2(28.6)$ & $2.13 \pm 4.16$ & $0.57 \pm 0.79$ & Hemangioma \\
\hline \multicolumn{7}{|l|}{$0.6 \mathrm{MeV}$} \\
\hline 12.5 cGy & 10 & 0 & 0 & 0 & 0 & \\
\hline 25 cGy & 16 & $6(37.5)$ & $5(31.3)$ & $4.18 \pm 7.03$ & $0.38 \pm 0.62$ & Harderian \\
\hline $50 \mathrm{cGy}$ & 22 & 10 (45.5), $\mathrm{P}=0.08$ & $9(40.1)^{\mathrm{a}}, \mathrm{P}=0.03$ & $1.23 \pm 2.19$ & $0.59 \pm 0.85$ & Hemangioma \\
\hline 100 cGy & 8 & $2 \quad(25)$ & $2 \quad(25)$ & $2.33 \pm 4.69$ & $0.38 \pm 0.74$ & \\
\hline
\end{tabular}

(Mean $\pm \mathrm{SD})$.

the $0.2 \mathrm{MeV}$ energy level, respectively. The figures were 0 , $5 / 22(22.7), 5 / 20(25 \%$, ovary tumor) and $1 / 9(11.1 \%$, ovary) at the $0.6 \mathrm{MeV}$ energy level (Table XII).

\section{Discussion}

The present experiments showed clear increase in the incidence of abnormal sperm in $\mathrm{C} 3 \mathrm{H}$ males following monoenergetic neutron irradiation, resulting in increased embryo lethality of $F_{1}$ offspring and liver tumors in surviving $F_{1}$ males. While the sperm abnormalities were energy dose-dependent, this did not appear to be the case for embryonic death and tumor incidence.
This lack of dose-dependence is in line with the literature. Inverse dose-dependence for fission spectrum neutron induction of somatic hprt deficiency mutations has been reported by Nakamura and Sawada (20) with mouse leukemia L5178Y cells and ${ }^{252} \mathrm{Cf}$-fission neutrons. Brenner and Hall published an inverse dose effect model for neoplastic transformation in vitro following high LET irradiation (21). Furthermore, Zhang et al (17) reported different doses of neutrons to produce approximately linear changes in the frequency of micronuclei in root-tip cells of Allium cepaonion irradiated as either dry dormant seeds or seedlings. Balcer-Kubiczek et al (22) earlier found modification of fission neutron doseresponse curves on varying the dose rate to be negligible or 
Table X. Body and organ weight for $\mathrm{F}_{1}$ female mice.

\begin{tabular}{|c|c|c|c|c|c|c|c|}
\hline Group & Body weight & Liver & Kidney & Ovary & Uterus & Adrenal & Spleen \\
\hline 0 cGy & $43.1 \pm 6.4$ & $1.60 \pm 0.28$ & $0.36 \pm 0.03$ & $0.026 \pm 0.023$ & $0.478 \pm 0.541$ & $0.026 \pm 0.023$ & $0.110 \pm 0.022$ \\
\hline \multicolumn{8}{|l|}{$0.2 \mathrm{MeV}$} \\
\hline $12.5 \mathrm{cGy}$ & $42.8 \pm 7.1$ & $1.63 \pm 0.22$ & $0.37 \pm 0.04$ & $0.022 \pm 0.005$ & $0.378 \pm 0.124$ & $0.022 \pm 0.005$ & $0.108 \pm 0.024$ \\
\hline $25 \mathrm{cGy}$ & $38.3 \pm 4.6^{\mathrm{b}}$ & $1.46 \pm 0.17$ & $0.36 \pm 0.04$ & $0.026 \pm 0.005$ & $0.580 \pm 0.472$ & $0.026 \pm 0.006$ & $0.110 \pm 0.030$ \\
\hline $50 \mathrm{cGy}$ & $44.4 \pm 9.3$ & $1.62 \pm 0.37$ & $0.38 \pm 0.04$ & $0.026 \pm 0.007$ & $0.509 \pm 0.176$ & $0.026 \pm 0.007$ & $0.120 \pm 0.054$ \\
\hline 100 cGy & $48.9 \pm 3.5^{b}$ & $1.62 \pm 0.23$ & $0.40 \pm 0.05$ & $0.070 \pm 0.138^{\mathrm{a}}$ & $0.299 \pm 0.160$ & $0.070 \pm 0.138^{\mathrm{a}}$ & $0.129 \pm 0.052$ \\
\hline \multicolumn{8}{|l|}{$0.6 \mathrm{MeV}$} \\
\hline $12.5 \mathrm{cGy}$ & $39.6 \pm 6.9$ & $1.58 \pm 0.30$ & $0.38 \pm 0.05$ & $0.029 \pm 0.010$ & $0.662 \pm 0.509$ & $0.028 \pm 0.009$ & $0.119 \pm 0.039$ \\
\hline $25 \mathrm{cGy}$ & $47.4 \pm 6.6$ & $1.77 \pm 0.30^{\mathrm{b}}$ & $0.40 \pm 0.06^{\mathrm{a}}$ & $0.033 \pm 0.033$ & $0.488 \pm 0.479$ & $0.033 \pm 0.033$ & $0.122 \pm 0.029$ \\
\hline $50 \mathrm{cGy}$ & $48.6 \pm 5.9^{b}$ & $1.95 \pm 0.33^{\mathrm{a}}$ & $0.44 \pm 0.04^{\mathrm{a}}$ & $0.021 \pm 0.006$ & $0.742 \pm 0.828$ & $0.021 \pm 0.006$ & $0.120 \pm 0.023$ \\
\hline 100 cGy & $46.3 \pm 9.1$ & $1.69 \pm 0.48$ & $0.37 \pm 0.08$ & $0.058 \pm 0.088$ & $0.256 \pm 0.171$ & $0.058 \pm 0.088$ & $0.103 \pm 0.042$ \\
\hline
\end{tabular}

$($ Mean $\pm \mathrm{SD})$. ${ }^{\mathrm{a} S i g n i f i c a n t l y ~ d i f f e r e n t ~ f r o m ~} 0 \mathrm{cGy}$ value $(\mathrm{P}<0.01)$; ${ }^{\mathrm{b}}$ Significantly different from 0 cGy value $(\mathrm{P}<0.05)$.

Table XI. Relative body weight for $\mathrm{F}_{1}$ female.

\begin{tabular}{|c|c|c|c|c|c|c|}
\hline Group & Liver & Kidney & Ovary & Uterus & Adrenal & Spleen \\
\hline 0 cGy & $37.3 \pm 5.4$ & $8.59 \pm 1.22$ & $0.606 \pm 0.495$ & $11.85 \pm 17.04$ & $0.237 \pm 0.186$ & $2.85 \pm 1.02$ \\
\hline \multicolumn{7}{|l|}{$0.2 \mathrm{MeV}$} \\
\hline $12.5 \mathrm{cGy}$ & $38.4 \pm 4.6$ & $8.88 \pm 1.09^{b}$ & $0.525 \pm 0.168$ & $9.16 \pm 3.75$ & $0.205 \pm 0.057^{\mathrm{b}}$ & $2.60 \pm 0.88$ \\
\hline $25 \mathrm{cGy}$ & $38.4 \pm 3.7$ & $9.43 \pm 1.15$ & $0.683 \pm 0.208$ & $15.47 \pm 12.24$ & $0.390 \pm 0.576$ & $2.88 \pm 0.77$ \\
\hline $50 \mathrm{cGy}$ & $35.0 \pm 6.4$ & $8.23 \pm 0.68$ & $0.553 \pm 0.152$ & $11.17 \pm 4.02$ & $0.202 \pm 0.054$ & $2.56 \pm 0.86$ \\
\hline 100 cGy & $33.1 \pm 3.0^{\mathrm{b}}$ & $8.15 \pm 0.90$ & $1.375 \pm 2.649^{\mathrm{b}}$ & $6.08 \pm 3.13$ & $0.197 \pm 0.050$ & $2.61 \pm 0.95$ \\
\hline \multicolumn{7}{|l|}{$0.6 \mathrm{MeV}$} \\
\hline $12.5 \mathrm{cGy}$ & $40.1 \pm 4.5$ & $9.74 \pm 1.45^{\mathrm{a}}$ & $0.727 \pm 0.234$ & $18.35 \pm 17.00$ & $0.267 \pm 0.078$ & $3.06 \pm 0.95$ \\
\hline $25 \mathrm{cGy}$ & $37.6 \pm 3.7$ & $8.52 \pm 1.41$ & $0.721 \pm 0.734$ & $10.71 \pm 10.57$ & $0.235 \pm 0.072$ & $2.62 \pm 0.71$ \\
\hline $50 \mathrm{cGy}$ & $40.1 \pm 3.4$ & $9.06 \pm 0.73$ & $0.445 \pm 0.134$ & $16.58 \pm 20.65$ & $0.171 \pm 0.027$ & $2.47 \pm 0.36$ \\
\hline 100 cGy & $36.4 \pm 5.3$ & $8.05 \pm 0.59$ & $1.188 \pm 1.756$ & $5.33 \pm 3.39$ & $0.192 \pm 0.055$ & $2.23 \pm 0.65$ \\
\hline
\end{tabular}

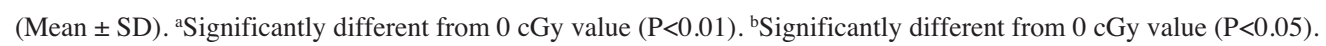

absent. On the other hand, Hill and Williams-Hill (23) observed that reduction of the dose rate of fission neutrons increases their effectiveness for transformation of C3H 10T1/ 2 cells. Watanabe et al (24) reported that a single ${ }^{252} \mathrm{Cf}$ neutron dose resulted in higher incidences of ovarian and Harderian gland tumors than the same total dose given at a low dose rate with $\mathrm{B} 6 \mathrm{C} 3 \mathrm{~F} 1$ mouse whole body irradiation. Clearly there may be differences between the in vitro and in vivo situations. It is considered that cells with large chromosomal aberrations or other abnormalities might be able to survive in vitro, but in vivo they might not, so smaller non-lethal chromosomal changes such as point mutations, frame shifts, as small additions or deletions could be essential for tumor induction in vivo. The source of irradiation, strain, sex, age and plants or animals are all clearly factors which need to be taken into account when determining radiation sensitivity. Recently, we reported that there were no significant differences in the tumor induction rate among the different energy such as $0.18,0.32,0.6$ and $1.0 \mathrm{MeV}$ monoenergetic neutron irradiation (18). Sasaki et al (25) also mentioned that induction of chromosome aberrations is not clearly dependent on neutron energy. In conclusion, there have been no consistent differences in tumor incidence among the various energies of neutron irradiation applied.

Goud et al (26) reported that exposure of mice to ${ }^{252} \mathrm{Cf}$ neutrons and gamma rays resulted in a decrease in testis weight and a concomitant increase in frequency of abnormal sperm. According to Hugenholtz and Bruce (19) X-ray- 
Table XII. Incidence of tumor for $\mathrm{F}_{1}$ female mice.

\begin{tabular}{rccl}
\hline Group & $\begin{array}{c}\text { Effective no. } \\
\text { of animal }\end{array}$ & $\begin{array}{c}\text { Positive } \\
(\%)\end{array}$ & \multicolumn{1}{c}{ Type of tumor } \\
\hline $0 \mathrm{cGy}$ & 64 & $3(4.7)$ & $\begin{array}{l}\text { Hemangioma, lymphoma, } \\
\text { ovary }\end{array}$ \\
& & & \\
$0.2 \mathrm{MeV}$ & & $3(13.0)$ & Hepatoma, lung, ovary \\
$12.5 \mathrm{cGy}$ & 23 & $3(13.6)$ & Ovary 3 \\
$25 \mathrm{cGy}$ & 22 & $1(5.0)$ & Ovary \\
$50 \mathrm{cGy}$ & 20 & 0 & \\
$100 \mathrm{cGy}$ & 9 & 0 & \\
$0.6 \mathrm{MeV}$ & & $5(22.7)$ & Hepatoma, lymphoma, \\
$12.5 \mathrm{cGy}$ & 19 & & $\begin{array}{l}\text { ovary 2, sarcoma } \\
25 \mathrm{cGy}\end{array}$ \\
& 22 & $5(25.0)$ & $\begin{array}{l}\text { Ovary } 5 \\
\text { Ovary }\end{array}$ \\
$50 \mathrm{cGy}$ & 20 & $1(11.1)$ & Ovary \\
\hline
\end{tabular}

induced abnormalities in sperm are transmissible up to the $\mathrm{F}_{2}$ generation as dominant mutations. Nomura $(27,28)$ demonstrated an increase in the dominant lethality and congenital malformations in offspring of male or female mice irradiated with X-rays (6) or treated with urethane $(27,28)$. These findings were further confirmed by Kirk and Lyon (29), West et al (30) and Lyon and Renshaw (31), using the same dose but different strains of mice. Nomura (6) also reported increased fetal death of $F_{1}$ offspring after paternal irradiation at the stage of spermatozoa and spermatids in a dose-dependent manner. Kurishita et al (32) demonstrated that external abnormalities are induced in offspring of male mice following treatment of germ cells at the spermatogonia stage with ${ }^{252} \mathrm{Cf}$ neutrons and the dose-response curve was linear up to 0.95 cGy. Streffer (33) similarly observed that a transgenerational transmission occurs for ionizing radiation-induced congenital malformations as well as for genomic instability, the latter measured at the chromosome level. Carls et al (34) described that ionizing radiation exposure of the germline can induce delayed DNA deletions in offspring mice. They suggested that DNA deletion events are implicated in the onset of carcinogenesis and a similar phenomenon in humans may account for a portion of childhood cancers. Nomura (6) found the incidence of tumors in $\mathrm{F}_{1}$ mice of the ICR strain to increased, in this case dose-dependently, after paternal exposure to 36,216 or $364 \mathrm{cGy}$ of X-rays at the stage of spermatozoa, spermatids or spermatogonia. Of the tumors occurring in the $\mathrm{F}_{1}$ offspring, $90 \%$ were lung tumors. Daher et al (35) reported that paternal X-ray irradiation resulted in reduction of litter size and a marginally significant doubling of the leukemia/lymphoma rate in the offspring in N5 strain mice, over a 1 year observation period. Urethane treatment of $F_{1}$ offspring derived from irradiated parents caused a 2.4 times greater incidence of tumors than observed in untreated controls (36). Vorobtsova et al (37) reported similar results with a different mouse strain. Mewissen et al (38) found that repeated administration of ${ }^{3} \mathrm{H}_{2} \mathrm{O}$ as the drinking water to C57BL/6M males before mating over several generations gave rise to hereditary adenocarcinomas in the small intestine. Essentially comparable effects of chemical carcinogens have been reported (39-41). A high incidence of liver tumors was observed in the $\mathrm{F}_{1}$ offspring of $\mathrm{C} 3 \mathrm{H}$ male mice which had been exposed to $50 \mathrm{cGy}$ of ${ }^{252} \mathrm{Cf}$ neutrons and mated with $\mathrm{C} 57 \mathrm{BL} / 6$ females $(8,9)$. In the present experiment, similar results were observed with $50 \mathrm{cGy}$ especially at the $0.6 \mathrm{MeV}$ energy level. Shay et al (42) documented that when 35- to 46-day-old Wistar rat females were treated with 3-methylcholanthrene using gastric tubes every day for two months and then mated with untreated males, the incidence of cancer was increased significantly in $\mathrm{F}_{1}$ and $\mathrm{F}_{2}$ offspring. Tomatis et al (5) subsequently found in the BDV1 rat system that the incidence of nerve tumors was significantly elevated in the $F_{1}$ generation when mating occurred two weeks after treatment of 9-week-old male rats with $80 \mathrm{mg} / \mathrm{kg}$ of ethylnitrosourea. Dasenbrock et al (43) described that maternal preconceptual exposure in C57BL/6J mice to radiation is associated with a moderately increased incidence of liver and lung tumors in the male descendants. The incidence of total tumors in the $F_{1}$ offspring, however, was not different from the control value. Lord et al (44) reported that with methylnitrosourea following preconceptional paternal contamination with ${ }^{239}$ plutonium the second generation excess of leukemia appears to be the result of preconceptional paternal irradiation and may be related to inherited changes that affect the development of haemopoietic stem cells. The evidence in humans is most derived from case reports and epidemiological studies of consequences to the progeny of paternal occupational exposure to chemicals, ionizing radiation and electromagnetic fields prior to conception $(3,45-47)$. Dasenbrock et al (43) indicated that maternal preconceptual X-ray exposure to radiation is associated with a moderately increased incidence of liver and lung tumors in male descendants in $\mathrm{C} 57 \mathrm{BL} / 6 \mathrm{~N}$ mice. Thus the fact that genetic damage to parental germ cells can be transmitted to the offspring as an origin of carcinogenesis has been well documented, and this was confirmed in the present experiment.

However, Cattanach et al (48) described no significant increase but seasonal changes in the incidence of lung tumors in offspring of $\mathrm{BALB} / \mathrm{cJ}$ or $\mathrm{C} 3 \mathrm{H} / \mathrm{Heh}$ mice exposed to X-rays following the experimental protocol of Nomura (6). Evidence for such seasonal changes in tumor incidence has been published and this relates to experiments carried out in insufficiently controlled animal facilities and experimental conditions, e.g., animals exposed to outdoor light. In fact, change of the light-dark interval significantly influences tumor frequencies in mice (49). Cattanach et al (48) also reported that reduction in litter size in paternally irradiated groups might be evidence of genetic damage, i.e., dominant lethality, resulting from the radiation exposure.

As a general rule, heavier mice are more likely to develop spontaneous and induced tumors earlier and caloric restriction decreases body weights and tumor incidences and increases longevity. Selby et al (50) suggested that induced dominant lethality in mice or rats with increased tumor rates have no relation with induction of dominant tumor mutations. In the 
present experiment numbers of offspring were lower with 100 cGy at both energy levels and the fact that only a few animals survived means that the incidence of liver tumors might not have been accurate. The range of gene damage is presumably very wide, given the sperm abnormalities and the embryo lethality and malformations, and many embryos died, so that surviving animals might have been those less susceptible to induction of tumors. However, if gene damage is limited, tumor-prone animals might survive, resulting in greater causation of tumors. Nomura suggested that germline exposure is a very early tumorigenesis by itself. It is possible that the lack of increase in lung tumors reported by Cattanach et al (48) may be attributable to increased incidence of embryo lethality caused by high doses of paternal $\mathrm{X}$-ray irradiation.

In conclusion, the results of the present study indicate that paternal exposure to radiation is associated with an increased incidence of liver tumors in the male descendants. While our study was not designed to investigate the mechanism of transmission of increased risk, the results are in keeping with the hypothesis of a germ line-transmitted hereditary effect of monoenergetic neutron irradiation.

\section{Acknowledgements}

We are grateful to Professor T. Nomura, Osaka University, and to Dr M.A. Moore for critical reading of this manuscript, and Mr. T. Nishioka for technical assistance.

\section{References}

1. Napalkov NP, Rice JM, Tomatis L and Yamasaki H: Perinatal and multigeneration carcinogenesis. IARC Scientific Publication, Lyon, 96, 1989.

2. Tomatis L, Narod S and Yamasaki H: Transgeneration transmission of carcinogenic risk. Carcinogenesis 13: 145-151, 1992.

3. Tomatis L: Transgeneration carcinogenesis: a review of the experimental and epidemiological evidence. Jpn J Cancer Res 85: 443-454, 1994

4. Gardner MJ, Snee MP, Hall AJ, Powell CA, Downes S and Terrell JD: Results of the case-control study of leukemia and lymphoma among young people near Sellafield nuclear plant in West Cumbria. Br Med J 300: 423-429, 1990.

5. Tomatis L, Cabral JRP, Likhachev AJ and Ponomrkov V: Increased cancer incidence in the progeny of male rats exposed to ethylnitrosourea before mating. Int J Cancer 28: 475-478, 1981.

6. Nomura T: Parental exposure to $\mathrm{X}$ rays and chemicals induces heritable tumors and anomalies in mice. Nature 296: 575-577, 1982.

7. Takahashi $\mathrm{T}$, Watanabe $\mathrm{H}$, Dohi $\mathrm{K}$ and Ito $\mathrm{A}:{ }^{252} \mathrm{Cf}$ relative biological effectiveness and inheritable effect of fission neutrons in mouse liver tumorigenesis. Cancer Res 52: 1948-1953, 1992.

8. Watanabe H, Takahashi T, Lee JY, et al: Influence of paternal ${ }^{252} \mathrm{Cf}$ neutron exposure on abnormal sperm, embryonal lethality, and liver tumorigenesis in the F1 offspring of mice. Jpn J Cancer Res 87: 51-57, 1996.

9. Shoji S, Masaoka Y, Kurosumi M, Katoh O and Watanabe H: Tumorigenesis in $\mathrm{F}_{1}$ offspring mice following paternal $12.5 \mathrm{cGy}$ ${ }^{252} \mathrm{Cf}$ fission neutron irradiation. Oncol Rep 5: 1175-1178, 1998 .

10. Miller RC, Marino SA, Martin SG, et al: Neutron-energydependent cell survival and oncogenic transformation. J Radiat Res (Tokyo) (Suppl) 40: 53-59, 1999.

11. Pandita TK and Geard CR: Chromosome aberrations in human fibroblasts induced by monoenergetic neutrons. I. Relative biological effectiveness. Radiat Res 145: 730-739, 1996.

12. Kubota N, Okada S, Nagatomo S, et al: Mutation induction and RBE of low energy neutrons in V79 cells. J Radiat Res (Tokyo) (Suppl) 40: 21-27, 1999.
13. Tanaka K, Gajendiran N, Endo S, Komatsu K, Hoshi M and Kamada N: Neutron energy-dependent initial DNA damage and chromosomal exchange. J Radiat Res (Tokyo) (Suppl) 40: 36-44, 1999.

14. Tanaka K, Kobayashi T, Sakurai Y, Nakagawa Y, Endo S, Hoshi M: Dose distributions in a human head phantom for neutron capture therapy using moderated neutrons from the 2.5 $\mathrm{MeV}$ proton- ${ }^{7} \mathrm{Li}$ reaction or from fission of ${ }^{235} \mathrm{U}$. Phys Med Biol 46: 2681-2695, 2001

15. Gajendiran N, Tanaka K and Kamada N: Comet assay to sense neutron 'fingerprint'. Mutat Res 452: 179-187, 2000.

16. Schmid E, Schlegel D, Guldbakke S, Kapsch RP and Regulla D: $\mathrm{RBE}$ of nearly monoenergetic neutrons at energies of $36 \mathrm{keV}$ 14.6 MeV for induction of dicentrics in human lymphocytes. Radiat Environ Biophys 42: 87-94, 2003.

17. Zhang W, Fujikawa K, Endo S, Ishikawa M, Ohtaki M, Ikeda H and Hoshi M: Energy-dependent RBE of neutrons to induce micronuclei in root-tip cells of Allium cepa onion irradiated as dry dormant seeds and seedlings. J Radiat Res (Tokyo) 44: 171-177, 2003

18. Watanabe H, Kashimoto N, Kajimura J, Ishikawa M and Kamiya K: Tumor induction by monoenergetic neutrons in B6C3F1 mice. J Radiat Res (Tokyo) 48: 205-210, 2007.

19. Hugenholtz AP and Bruce WR: Radiation induction of mutations affecting sperm morphology in mice. Mutat Res 107: 177-185, 1983.

20. Nakamura N and Sawada S: Reversed dose-rate effect of RBE of ${ }^{252}$ calfornium radiation in the induction of 6-thioguanineresistant mutations in mouse L5176Y cells. Mutat Res 201: 65-71, 1988.

21. Brenner DJ and Hall EJ: The inverse dose-rate effect for oncogenic transformation by neutrons and charged particles: a plausible interpretation consistent with published data. J Radiat Biol 58: 745-758, 1990.

22. Balcer-Kubiczek EK, Harrison GH, Hill CK and Blakely WF: Effects of WR-1065 and WR-151326 on survival and neoplastic transformation in $\mathrm{C} 3 \mathrm{H} / 10 \mathrm{~T} 1 / 2$ cells exposed to TRIGA or JANUS fission neutrons. Int J Radiat Biol 63: 37-46, 1993.

23. Hill CK and Williams-Hill D: Neutron carcinogenesis: past, present and future. J Radiat Res (Tokyo) (Suppl) 40: 117-127, 1999.

24. Watanabe H, Okamoto T, Yamada K, et al: Effects of dose rate and energy level on fission neutron $\left({ }^{252} \mathrm{Cf}\right)$ tumorigenesis in B6C3F1 mice. J Radiat Res (Tokyo) 34: 235-239, 1993.

25. Sasaki MS, Endo S, Ejima Y, et al: Effective dose of A-bomb radiation in Hiroshima and Nagasaki as assessed by chromosomal effectiveness of spectrum energy photons and neutrons. Radiat Environ Biophys 45: 79-91, 2006.

26. Goud SN, Feola JM and Maruyama Y: Sperm shape abnormalities in mice exposed to californium-252 radiation. Int $\mathrm{J}$ Radiat Biol 52: 755-760, 1987.

27. Nomura T: Transmission of tumors and malformations to the next generation of mice subsequent to urethan treatment. Cancer Res 35: 264-266, 1975.

28. Nomura T: Transgenerational effects from exposure to environmental toxic substances. Mutat Res 659: 185-193, 2008.

29. Kirk M and Lyon MF: Induction of congenital malformations in the offspring of male mice treated with X-rays at pre-meitic and post-meiotic stages. Mutat Res 125: 75-85, 1984.

30. West JD, Kirk Y, Goyder Y and Lyon MF: Discrimination between the effects of X-ray irradiation of the mouse oocyte and uterus on the induction of dominant lethals and congenital anomalies. I. Embryo-transfer experiments. Mutat Res 149: 221-230, 1985.

31. Lyon MF and Renshaw R: Induction of congenital malformations in mice by parental irradiation: transmission to later generations. Mutat Res 198: 277-283, 1988.

32. Kurishita A, Ono T, Okada S, Mori Y and Sawada S: Induction of external abnormalities in offspring of male mice irradiated with ${ }^{252} \mathrm{Cf}$ neutron. Mutat Res 268: 323-328, 1992.

33. Streffer C: Transgenerational transmission of radiation damage: genomic instability and congenital malformation. J Radiat Res (Tokyo) (Suppl B) 47: B19-B24, 2006.

34. Carls Nand Schiestl RH: Effect of ionizing radiation on transgenerational appearance of $\mathrm{p}(\mathrm{un})$ reversions in mice. Carcinogenesis 20: 2351-2354, 1999.

35. Daher A, Varin M, Lamontagne Y and Oth D: Effect of preconceptional external or internal irradiation of N5 male mice and the risk of leukemia in their offspring. Carcinogenesis 19: 1553-1558, 1998 
36. Nomura T: X-ray-induced germ-line mutation leading to tumors. Its manifestation in mice given urethane post-natally. Mutat Res 121: 59-65, 1983.

37. Vorobtsova IE and Kitaev EM: Urethane-induced lung adenomas in the first-generation progeny of irradiated male mice. Carcinogenesis 11: 1931-1934, 1988.

38. Mewissen J, Ugarte AS and Rust JH: Tumeur intestinale hereditaire observé après irradiation de generations multiples d'une lignee germinale male de la souris C57BL/6. CR Soc Biol 178: 230-235, 1984.

39. Strong LC: Genetic analysis of the induction of tumors by methylcholanthrene. Am J Cancer Inst 39: 347-349, 1940.

40. Strong LC: Genetic analysis of the induction of tumors by methylcholanthrene. IX. Induced and spontaneous adenocarcinomas of the stomach in mice. J Natl Cancer Inst 5: 339-362, 1940 .

41. Boutwell RK: Some biological aspects of skin carcinogenesis. Prog Exp Tumor Res 4: 207-250, 1964.

42. Shay H, Gruenstein $M$ and Weinberger M: Tumor incidence in $F_{1}$ and $F_{2}$ generations derived from female rats fed methylcholanthrene by stomach tube prior to conception. Cancer Res 12: 296,1952 .

43. Dasenbrock C, Tillmann T, Ernst H, et al: Maternal effects and cancer risk in the progeny of mice exposed to X-rays before conception. Exp Toxicol Path 56: 351-360, 2005.
44. Lord BI, Woolford LB, Wang L, et al: Induction of lymphohaemopoietic malignancy: impact of preconception paternal irradiation. Int J Radiat Biol 74: 721-728, 1998.

45. Savitz DA and Chen JH: Parental occupation and childhood cancer: review of epidemiologic studies. Env Health Per 88: 325-337, 1990

46. O'Leary LM, Hicks AM, Peters JM and London S: Parental occupational exposures and risk of childhood cancer: a review. Am J Ind Med 20: 17-35, 1991.

47. Pearce MS, Hammal DM, Dorak MT, McNally RJ and Parker L: Paternal occupational exposure to electro-magnetic fields as a risk factor for cancer in children and young adults: a casecontrol study from the North of England. Ped Blood Cancer 49: 280-286, 2007.

48. Cattanach BM, Patrick G, Papworth D, et al: Investigation of lung tumour induction in BALB/cJ mice following paternal X-irradiation. Int J Radiat Biol 67: 607-615, 1995.

49. Nakajima H, Narama I, Matsuura T and Nomura T: Enhancement of tumor growth under short light/dark cycle in mouse lung. Cancer Lett 78: 127-131, 1994.

50. Selby PB, Earhart VS and Raymer GD: The influence of dominant lethal mutations on litter size and body weight and the consequent impact on transgenerational carcinogenesis. Mutat Res 578: 382-394, 2005 . 